\title{
Imitation of honey bees' concept learning processes using Vector Symbolic Architectures
}

\author{
Denis Kleyko, ${ }^{a, *}$, Evgeny Osipov ${ }^{\mathrm{a}}$, Ross W. Gayler ${ }^{\mathrm{b}}$, Asad I. Khan ${ }^{\mathrm{c}}$, Adrian G. Dyer ${ }^{\mathrm{d}}$ \\ ${ }^{a}$ Department of Computer Science Electrical and Space Engineering, Luleå University of Technology, 971 \\ 87 Luleå, Sweden \\ ${ }^{b}$ Independent Researcher, Melbourne, VIC, Australia \\ ${ }^{c}$ Clayton School of Information Technology, Monash University, Clayton, VIC, Australia \\ ${ }^{d}$ Media and Communication School, Royal Melbourne Institute of Technology, Melbourne, VIC, Australia
}

\begin{abstract}
This article presents a proof-of-concept validation of the use of Vector Symbolic Architectures as central component of an online learning architectures. It is demonstrated that Vector Symbolic Architectures enable the structured combination of features/relations that have been detected by a perceptual circuitry and allow such relations to be applied to novel structures without requiring the massive training needed for classical neural networks that depend on trainable connections.

The system is showcased through the functional imitation of concept learning in honey bees. Data from real-world experiments with honey bees (Avarguès-Weber et al., 2012) are used for benchmarking. It is demonstrated that the proposed pipeline features a similar learning curve and accuracy of generalization to that observed for the living bees. The main claim of this article is that there is a class of simple artificial systems that reproduce the learning behaviors of certain living organisms without requiring the implementation of computationally intensive cognitive architectures. Consequently, it is possible in some cases to implement rather advanced cognitive behavior using simple techniques.
\end{abstract}

Keywords: Vector Symbolic Architecture, hyperdimensional computing, distributed data representation, concept learning, cognition, inference.

\section{Introduction}

Over the past two decades the honeybee has become established as an important model system in the study of machine vision (Srinivasan, 2006, 2010, 2011), and recent insights into cognition in bee brains have provided a potentially useful model

\footnotetext{
* Corresponding author

Email addresses: denis.kleyko@ltu.se (Denis Kleyko), evgeny.osipov@ltu.se (Evgeny Osipov), r.gayleregmail.com (Ross W. Gayler), asad.khan@monash.edu (Asad I. Khan), adrian.dyerermit. edu. au (Adrian G. Dyer)
} 
that could be used to enable efficient learning by machine vision systems. For example, behavioral experiments on free flying honeybees have shown that these insects estimate distance traveled by measuring the optic flow of spatial information content (Srinivasan, 2010), a principle that has since been incorporated into flying machines (Srinivasan, 2011; Soccol et al., 2007).

This article describes the design of an artificial learning system that imitates the concept learning process of a living organism. The approach is inspired by experiments on honeybees that revealed these insects' abilities to learn new concepts (Giurfa et al., 2001; Avarguès-Weber et al., 2012, 2014). In particular, it draws on a study in which bees were placed in a maze of the sort shown in Figure 3 and trained to select one direction over the other. This impressive learning process requires the bees to recognize a specific visual input (a pattern formed from two 2D geometrical shapes with specific attributes arranged in a fixed order on a plane) and associate it with a reward (access to sucrose solution); flying in the other direction resulted in a penalty (the bee was offered a quinine solution). The behavioral experiments were designed to exploit reinforcement learning during the training phase.

One of the major challenges when attempting to imitate this learning process (AvarguèsWeber et al., 2012) with a computer is that the learning system must achieve its goal using a very small data set (only 30 training runs were performed in the behavioral experiments) and be able to incrementally update the learned concept to provide flexibility in the interpretation of previously unseen visual targets.

\subsection{Contribution of the article}

This article focuses on the proof-of-concept validation of the applicability of Vector Symbolic Architectures (VSA) for imitating the learning behavior of living organisms. To this end, the first VSA-based system for the functional modeling of (part of) the concept learning process of honeybees was developed and evaluated. The proposed architecture approximates the functional behavior of bees without mapping it to beespecific mechanisms/anatomy. It has previously been shown (Eliasmith, 2013) that VSAs are relatively easy to implement neurally; therefore, given the success of our VSA-based system, it may be interesting to re-investigate bees' learning processes to see whether they exhibit characteristics typical of VSA-based processing.

The work presented in this article can be considered bio-inspired in three respects. First, its main aim was to model aspects of the cognitive behavior of a living organism, i.e. the experimentally demonstrated concept learning process of honey bees. Second, the techniques used to model this behavior (i.e. distributed representations and vector symbolic architectures) are both bio-inspired in that they have been shown to mirror the behavior of neurons in the human brain when representing and reasoning based on structured knowledge. In particular, the simplest possible implementation of Kanerva's "learning by example" (Kanerva, 2000) is adopted in this work. Finally, the online concept learning architecture is anchored to a psychologically-plausible model for similarity analysis, i.e. the hybrid model of structural, spatial and featural similarity. It is demonstrated that the proposed system has many characteristics in common with the bees' learning system: it requires only a few training trials, it is able to continuously update learned concepts, and it exhibits flexibility in the interpretation of previously unseen visual stimuli. 
In brief, the key features of the work presented herein are that:

- Vector symbolic architectures were used to represent structured combinations of features/relations and to apply these relations to novel structures in the online learning context;

- The choice of relation representation was motivated by classical analogical similarity models;

- The VSA representations were used as the central component of a learning pipeline with a perceptual circuitry and made it possible to apply the represented relations to novel structures without the massive training needed for classical neural networks;

- Proof-of-concept validation of the proposed online learning pipeline was performed by using it to reproduce the concept learning capabilities of honeybees.

\subsection{Limitations}

Although the aim of this work was to develop a generic architecture for online concept learning, some of the design choices were tailored to the specifics of the considered biological experiments. Specifically, the developed system was designed to analyze simple two dimensional visual stimuli consisting of a pair of objects and their relationship to one-another. However, it seems likely that the proposed approach would also be relevant for organisms with higher cognitive capabilities, i.e. the ability to process more complex scenes. In particular, Miller (1956) famously showed that there is an upper bound on the number of items ("chunks") that humans can process simultaneously. It is thus clear that even very complex scenes can be modeled using a finite number of parameters. It is likely that the real challenge to overcome in such cases is in identifying the optimal chunks with which to represent the situation at a given point in time.

\subsection{Structure of the article}

The article is structured as follows. Section 2 presents an overview of related work relevant to the article's contents. Section 3 presents a conceptual overview of the proposed learning system and describes the results of several experiments on honey bees that were used as sources of reference data with which to showcase the system. Section 4 outlines the theory of Vector Symbolic Architectures and demonstrates their applicability to the problem addressed in the article. The details of the design of the artificial learning system are then presented in Section 5. The learning performance of the new system is evaluated in Section 6. Finally, the conclusions and some suggestions for future work are presented in Section 7.

\section{Related Work}

The first clear evidence that honeybees can learn visual stimuli and relate them to rules was presented by Giurfa et al. (2001), who showed that honeybees can learn 
concepts of sameness and difference relating to visual stimuli via a process of delayed matching. In a typical experiment, an individual bee had to view a given target stimulus (e.g a yellow disc), load this information into its working memory, and subsequently use the information to choose between two alternatives (e.g. yellow disc versus blue disc). The bees had to learn to use rules for matching test stimuli to samples because the nature of the visual stimuli was changed continuously during the experiments. Importantly, once the bees had learned rules relating to the visual domain, they were able to apply these rules to novel stimuli presented in the olfactory domain. The bees were also able to learn the opposite rule (i.e., do not match the sample) and thus demonstrated a remarkable degree of plasticity in learning for problem solving.

Later, Avarguès-Weber et al. (2011) showed that bees are capable of learning the above-below relation in the context of visual stimuli, and of applying learned relations of this sort to novel stimuli in transfer tests. Experiments conducted by AvarguèsWeber et al. (2012) showing that honeybees can learn and apply two concepts simultaneously - specifically, the bees were able to combine a sameness-difference rule with either an above-below or a left-right rule, applying each rule to separate components of a given stimulus. Importantly, individual bees could simultaneously process both levels of such rule-based problems even though only a single level might function as a reward predictor, suggesting that bee cognition has evolved to acquire the maximum possible information content from relevant stimuli. It is likely that such impressive learning capabilities result from the allocation of attention to various components of stimuli, since bees receiving different levels of priming to either local or global information content within a complex scene modulate their choices according to the most relevant components Avarguès-Weber et al. (2015).

An overview of major achievements in the study of conceptual learning by miniature brains has been presented by Avarguès-Weber et al. (2013). More recent work (Avarguès-Weber et al., 2014) has shown that honeybees can discriminate and learn relations based on the relative sizes of objects in their field of view, and that having learned such rules, bees can apply them to novel stimuli with different shapes, sizes, and colors. The potential applicability of these findings in computer vision has been discussed by Dyer et al. (2014), who emphasized that insights gleaned by studying evolved insect systems can be tremendously helpful when designing software for machine vision systems because they can highlight the most useful sensory channels for solving complex problems. An important conclusion is that rule learning could be a useful ability for computer vision systems because learned rules can be applied to novel previously unseen scenarios. A similar discussion about concept learning in neuromorphic vision systems was published by Sandin et al. (2014), who considered the potential advantages of combining different techniques and results, such as neuromorphic systems, findings from studies on the capabilities of miniature (insect) brains, distributed computer vision systems, wireless data transmission and Vector Symbolic Architectures. Their paper established an important framework that could potentially be used to model the cognitive behaviors of real brains (such as those of honeybees). However, the practical implementation of such complex bio-like systems that can learn through experience has not yet been attempted to our knowledge.

Distributed data representation is widely used for computer based semantic reasoning (Kanerva, 2009; Plate, 2003). The cognitive capabilities achievable using dis- 
tributed representations have been demonstrated by creating systems capable of solving Raven's progressive matrices (Rasmussen and Eliasmith, 2011; Emruli et al., 2013; Levy et al., 2014). Vector Symbolic Architectures (VSAs) are bio-inspired representations of structured knowledge. Their development was stimulated by studies on brain activity that showed that the processing of even simple mental events involves simultaneous activity in many dispersed neurons Kanerva (2009). Information in VSAs is similarly represented in a distributed fashion: a single concept is associated with a pattern of activation of many neurons. This is achieved by using codewords with very large dimensions. Several different types of VSAs have been introduced, each using different representations (Plate, 2003; Kanerva, 2009; Gayler, 1998; Rachkovskij and Kussul, 2001; Aerts et al., 2009; Rachkovskij et al., 2013; Gallant and Okaywe, 2013; Snaider and Franklin, 2014).

It was recently shown (Levy et al., 2014) that VSAs can solve three central problems identified by Wittgenstein: rule-following, aspect-seeing, and the development of a "private" language. Moreover, VSAs have been applied in a wide range of contexts. For example, Gallant and Okaywe (2013) proposed the use of a VSA-based knowledge-representation architecture called MBAT in machine learning algorithms. More recently, VSA-based systems for the analysis of generic patterns and symbol sequences have been proposed by Kleyko et al. (2015a), Kleyko and Osipov (2014a), Kleyko and Osipov (2014b), and Kleyko et al. (2015b). Räsänen and Kakouros (2014) present a VSA-based approach for modeling dependencies in multiple parallel data streams. Finally, VSAs were also used in the development of an architecture that achieves interoperability of systems with minimal prior configuration (Emruli et al., 2014).

This article utilizes a subclass of VSAs based on so-called Binary Spatter Codes (Kanerva, 1997). The approach adopted herein is somewhat reminiscent of that presented by Levy et al. (2013), who proposed a VSA-based knowledge-representation architecture capable of handling arbitrarily complex, hierarchical, symbolic relations (patterns) between sensors and actuators in robotics. However, whereas Levy's work showcased the representation of preprogrammed relations between sensors and actuators, this work uses VSA-based representations to enable learning from the observed environment.

\section{Outline of the proposed online concept learning architecture}

This work proposes a simple but extensible, online learning architecture that imitates the recently observed concept learning process in honeybees Avarguès-Weber et al. (2012). The proposed VSA-based pipeline is illustrated in Figure 1. The novel part of this architecture, and the primary contribution of this article, is the functionality of the stimuli encoding block, which is used to represent the observed episode as a set of relations between different objects. The construction of the stimuli encoding block is outlined in Figure 2.

The stimuli encoding block takes advantage of two useful properties of VSAs. The first of these is their ability to represent relationships between arbitrary, novel entities without requiring training. This representational capability arises directly from the 
structure of the VSA network and eliminates the need for extensive training using techniques such as backpropagation. Second, VSAs allow the representation of multiple relationships simultaneously on the same set of neurons, once again without specific training. These two properties enable the neural network to manage novel combinations of relationships with no need for extensive training.

The core of the stimuli encoding block is the pool of relations' representations or templates, which is described using VSAs. VSAs are described in detail in the next section; for our current purposes, it is sufficient to say that VSAs are a class of connectionist models for representing structured knowledge using (for example) binary codewords of very high dimensionality, i.e. thousands of bits. Throughout this article, the terms $H D$-codes, $H D$-vectors and $H D$-codewords are used interchangeably when referring to the codewords of the VSA class Kanerva (1997). Knowledge structuring is expressed by applying basic arithmetic operations to the codewords, which encode the concepts. Reasoning and inference-making are made possible by the unique statistical properties of long codewords Kanerva (2009), Plate (2003).

It is assumed that the system operates on episodes of visual inputs, where an episode is all of the visual input acquired during one learning cycle. Each learning cycle begins with the presentation of a visual training sample to the system, and ends with the presentation of a positive or a negative reward. During each episode, vision and feature extraction circuitries attempt to isolate entities in the environment, detect binary relations between those entities, and return vectors encoding the identities of the entities, the identity of the relationship between them, and the roles played by each entity in the relationship. Obviously, the system relies heavily on the capabilities of the visual circuitry, but this is true for all vision-based learning systems. The question of interest here is not how much is done by the specialized visual circuitry, but how to combine the outputs of the visual circuitry with generated rule-following behavior. VSAs are a powerful tool for this purpose.

All representations generated by the pipeline are used to encode relation representations (both spatial and comparative), which are are stored in the item memory. These are then encoded into two different forms of VSAs representation: the bonding representation, which captures the relationship between the entities, and the comparative representation, which captures the relationships between their features. The bonding representation is denoted as:

$$
\operatorname{bond}_{i}\left(r e_{i}, e_{i, 1}, e_{i, 2}\right)=\left[r e_{i}+r o_{1}\left(r e_{i}\right) \oplus e_{i, 1}+r o_{2}\left(r e_{i}\right) \oplus e_{i, 2}\right]
$$

The following notation is used for arithmetic operations in all of the equations presented in this manuscript. $\oplus$ denotes the bitwise XOR operation ${ }^{1}$. The notation $[A+B+C]$ is used for "majority summation" (also explained in the next section), i.e. the operation used to implement vector bundling in VSAs.

The names of the variables and functions used in (1) and (2) are mnemonic to facilitate understanding. Thus in (1) all the arguments of bond are HD-vectors supplied

\footnotetext{
${ }^{1}$ While the symbol $\otimes$ sign is used widely in the VSA- related literature to denote the operation of vector binding, we have chosen to use $\oplus$ to reflect the actual arithmetic operation used to implement binding and to avoid possible confusion with the tensor multiplication operation.
} 
by the visual and feature extraction circuitry. The subscript $i$ indicates the episode being processed by the visual circuitry. The HD-vector $r e$ indicates the identity of the binary relation detected by the visual circuitry. This is one of a small number of discrete values (codewords) used to indicate the relations that can be detected by the visual circuitry. Similarly, the ro functions $\left(\mathrm{ro}_{1}\right.$ and $\left.\mathrm{ro}_{2}\right)$ return values that are equal to the codewords representing each of the two roles of the binary relation represented by $r e$. Conceptually, the HD-vectors $r e, \mathrm{ro}_{1}(\mathrm{re}), \mathrm{ro}_{2}(\mathrm{re})$ should be generated by the visual circuitry, but treating their generation as a part of the representation makes it easier to understand the purpose of the VSA representation.)

The bonding representation can for example be used to encode a spatial relation between a pair of objects. For instance, for the the vertical alignment spatial relation labeled "above-below", the roles might be labeled "upper-entity" and "lower-entity". Note that these labels are chosen purely for expository convenience. Similarly, for the horizontal alignment relation labeled "left-right", the roles might be labeled "rightentity" and "left-entity". The actual values are arbitrary HD-vectors.

The comparative representation is denoted as:

$$
\operatorname{comp}_{i}\left(f, e_{i, 1}, e_{i, 2}\right)=\left[\operatorname{comprel}(f) \oplus \operatorname{comparison}_{f}\left(\text { feat }_{f}\left(e_{i, 1}\right), \text { feat }_{f}\left(e_{i, 2}\right)\right)\right]
$$

In (2) all the arguments of $\operatorname{comp}()$ are HD-vectors supplied by the visual and feature extraction circuitry. The subscript $i$ denotes the episode being processed by the visual circuitries. The function comprel $(f)$ returns the HD-vector that indicates the identity of the binary relation that is appropriate for comparison of values of feature $f$. The $f_{e a t}$ functions return the value of the feature $f$ for the argument entity $e$. For example, for $f=$ shape the returned values might be circle or square. The comparison $_{f}$ function returns the HD-vector representing the result of comparing two values of feature $f$. For example, for $f=$ shape the comparison of all possible shape value pairs might yield same or different. Again, these labels are purely for expository convenience. The actual values are arbitrary HD-vectors.

Representations (1) and (2) should be viewed as templates in the sense that at the time of the system initialization no particular meaning is given to the specific representation, and the objects that will be related are also not known. In other words the system starts in a "clean slate" state. In the remainder of the article, the terms representation and template are used interchangeably.

Honeybees served as the model for this study. While the full range of honeybees' rule learning capacities is yet to be determined, experiments have shown (AvarguèsWeber et al., 2011, 2012, 2014) that they are capable of processing at least the following relations: sameness-difference, above-below, left-right, and larger-smaller. Note that sameness-difference is defined with respect to specific properties of the images and is, therefore, the relation across any pair of attributes. The pipeline outlined above is not, however, limited in the number of potentially recognizable relations. The number of recognizable relations is determined by the complexity of the sensory and feature extraction systems on the one hand, and the size of the memory and computational power of the processing unit on the other, as might be expected given the wide range of intelligence levels among living organisms.

Once an episode has been processed, i.e. all of the entities and their relations have been processed and encoded into bonding and comparative representations, a complete 


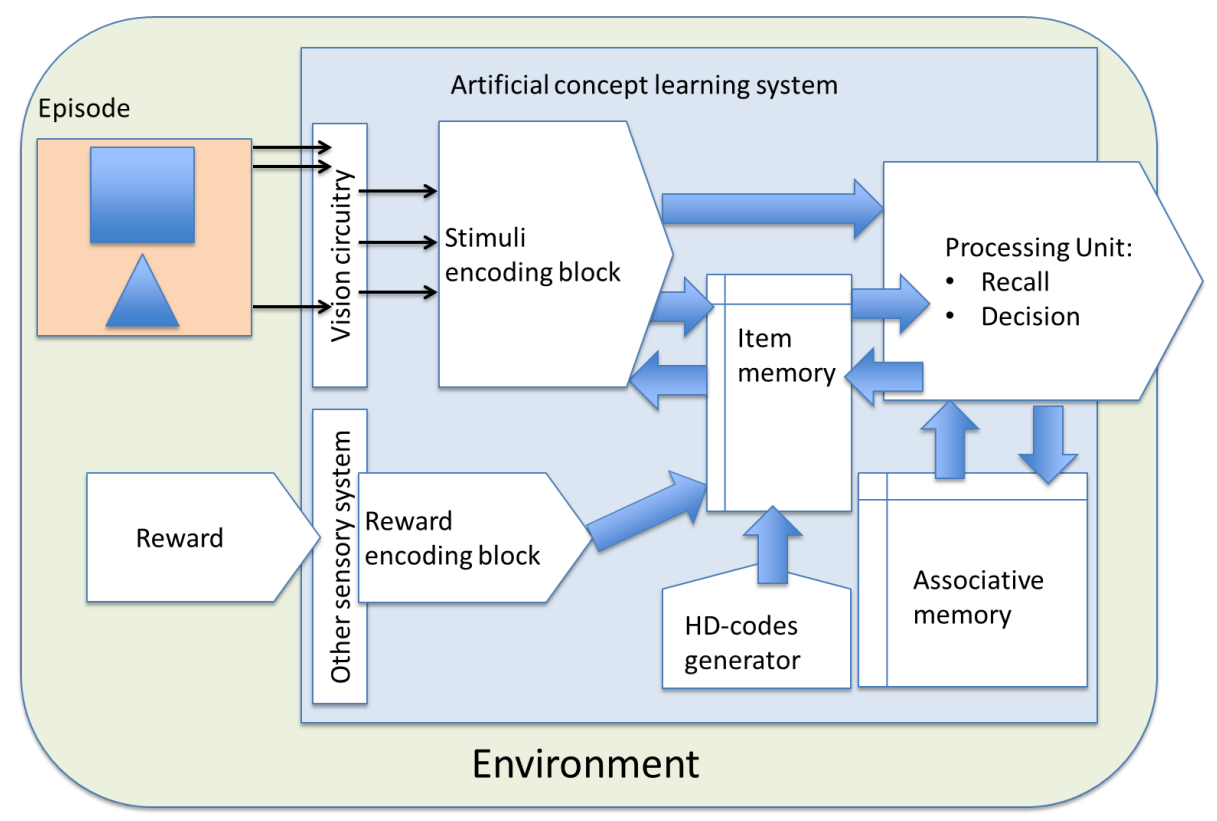

Figure 1: A simple concept learning architecture illustrating the interactions of the stimuli encoding block with the item memory, associative memory, recall, and processing units.

representation of the episode is constructed by bundling all the encoded representations as follows:

$$
\text { EPISODE }_{i}=\left[\operatorname{bond}_{i}(\ldots)+\operatorname{comp}_{i}(\ldots)\right]
$$

For example, assume an episode consists of two vertically aligned triangles (T1 and T2) of the same color but different sizes (sizeof $(T 1)<\operatorname{sizeof}(T 2)$ ). The bonding spatial representation for this episode is then encoded (with mnemonic labels) as bond $($ above - below, $T 1, T 2)=[$ above - below + above $\oplus T 1+$ below $\oplus T 2]$, while the comparative representation is encoded as $\operatorname{comp}(\operatorname{size}, T 1, T 2)=\operatorname{size} \oplus$ different. The episode as a whole is thus represented as EPISODE $=[$ bond (abovebelow, T1,T2) + comp $($ size, $T 1, T 2)]$.

The episode's representation (3) is then used by the processing unit for experience formation during the training phase, and also for the recall operation and decision making during the operating phase. To complete the process and represent the system's experience, the processing unit awaits the arrival of the reward signal. Upon receipt of the reward signal the processing unit binds it with the representation of the episode (e.g. EPISODE $\oplus$ REWARD) and updates the associative memory. During the system's lifetime, this experience is used in online reasoning and decision making processes. The detailed procedure is described in Section 5.

\subsection{The showcase: Description of the experiments with honeybees}

The proposed architecture for artificial concept learning is inspired by the experiments on free flying honeybees reported by Avarguès-Weber et al. (2012). The re- 


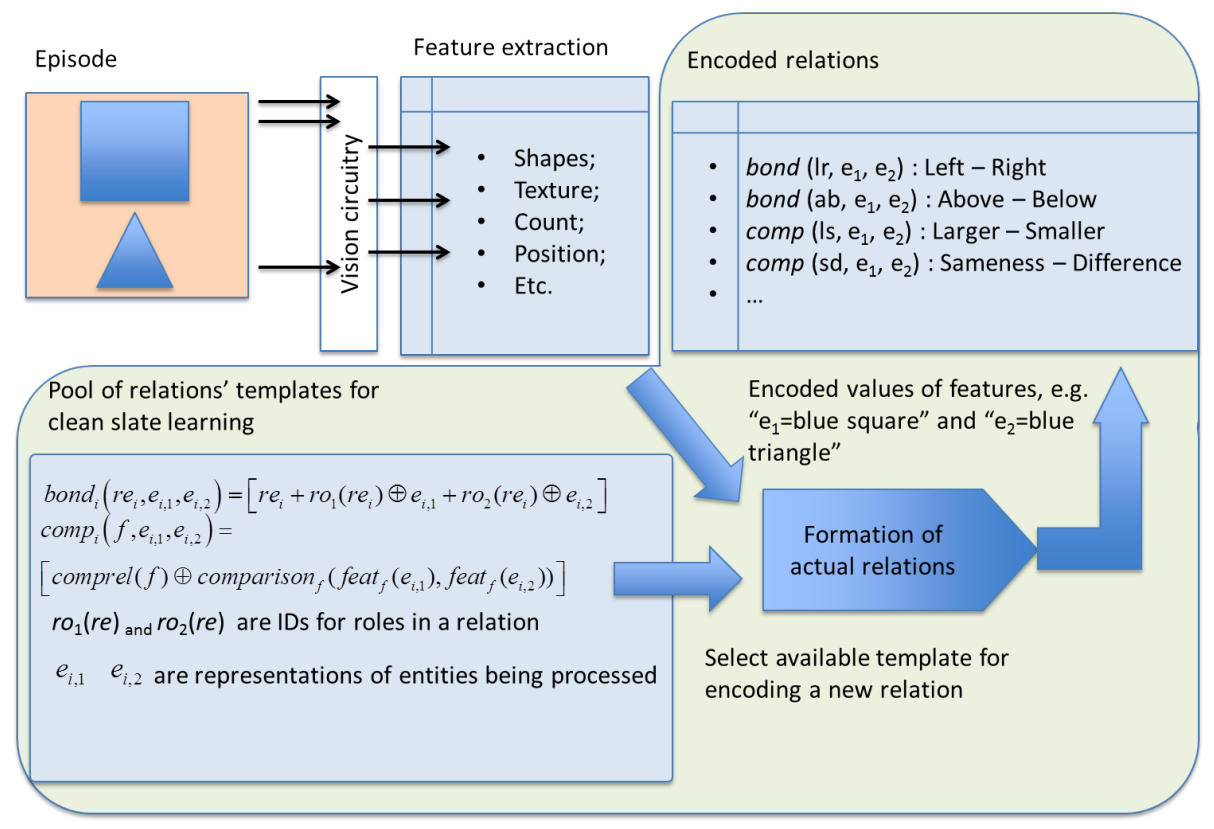

Figure 2: Details of the stimuli encoding block. The visual episode in this case consists of a square shape above and a triangle shape below.

A

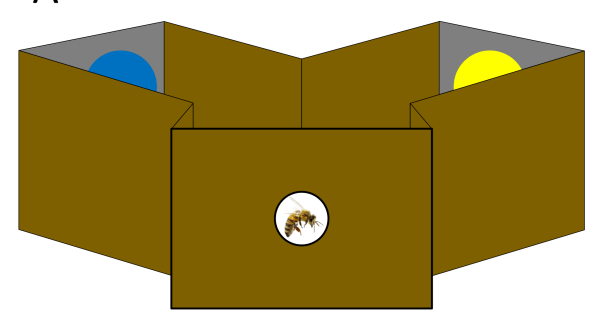

B

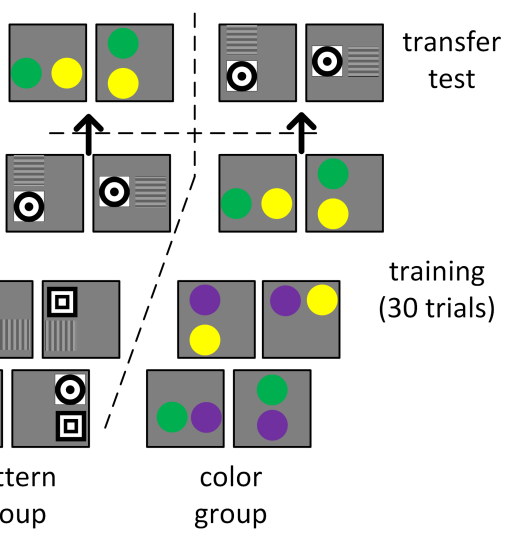

Figure 3: A. The maze used in the honeybee experiments. B. The two types of stimuli used in the honeybee experiments: pattern groups and colour groups. The bees had to choose between one of two visual stimuli in each case, applying either above-below or left-right relations to obtain a reward. Incorrect application of the relation resulted in a penalty, in the form of exposure to a quinine solution.

sults of these experiments were also used to benchmark the proposed solution's performance. This subsection summarizes the key findings of the honey bee training experiments. Their general setup is presented in Figure 3. The bees were trained to fly 

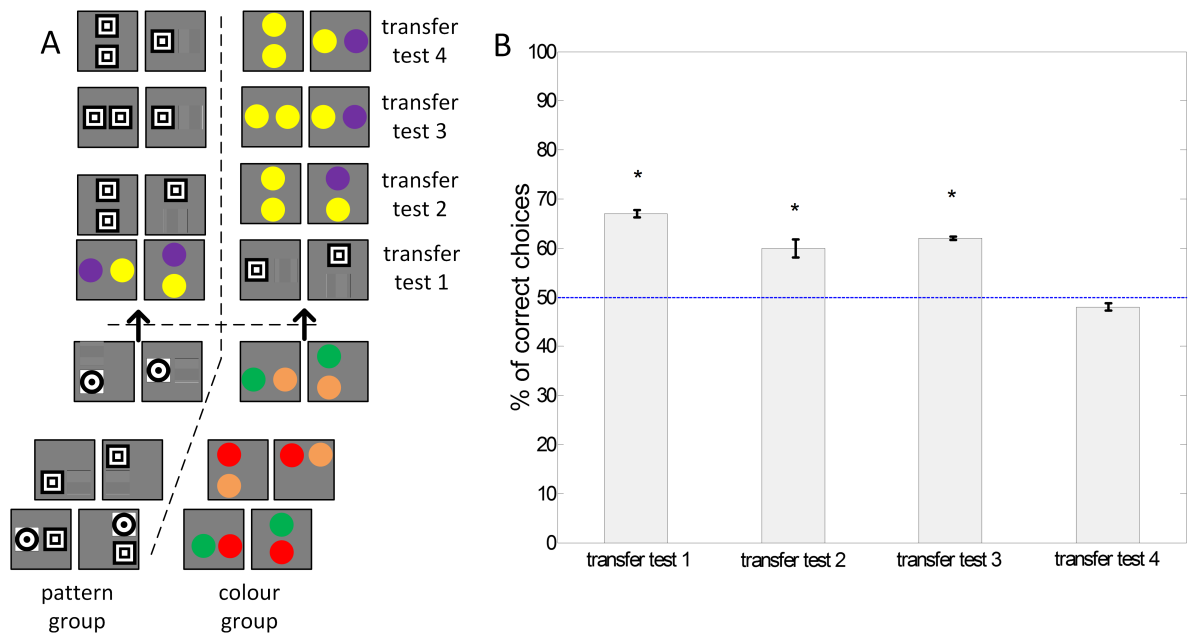

Figure 4: A. Bees were trained in a maze to choose between stimuli presenting two different patterns (group 1) or two differently colored discs (group 2) in an above-below (or right-left) relation depending on the group of bees. The results of transfer tests for the above-below relation are shown. B. Four transfer tests used to assess learned concepts. Thirty trials were performed for each test. In transfer test 1, correct choices correspond to the selection of the spatial relation that had previously yielded a reward; in transfer tests 2,3 and 4 , the term refers to the selection of the appropriate stimulus when presented with 2 different images. In transfer test 1 , bees transferred their choice to unknown stimuli that presented the same spatial relation even though the stimuli belonged to a different sensory modality. Transfer tests $2-4$ demonstrated that bees also learned that the stimuli had to present two different images. Asterisks $\left(^{*}\right)$ indicate cases where the percentage of correct choices was significantly different from 50\%. (C2013, Proceedings of the Royal Society B: Biological Sciences, The Royal Society, adopted with permission from Avarguès-Weber et al. (2013).

into a Y-shaped maze, which presented two alternative paths; the bees were required to choose either the left- or the right-hand path. Each path is marked with a different visual stimulus; one path leads to a reward (a sucrose solution) while the other leads to penalty (a quinine solution). The bees are motivated to collect as much of the reward as possible, and to avoid the quinine Avarguès-Weber et al. (2010). Consequently, the bee's learning objective is to correctly infer the path leading to sucrose solution each time it enters a maze.

Examples of the visual stimuli presented to bees are given in Figure 3. A visual stimulus in these experiments embodies two concepts that can be learned. One represents a spatial relation between shapes (above-below or left-right). The second learned concept sameness-difference relates to the features of the shapes in the episode, which consisted of different coloration or different hatching. The honey bees were put through 30 training trials for each type of stimulus, e.g. the achromatic shapes. Potential bias in the bees' selections was avoided by varying which side of the maze offered the rewarded and non-rewarded stimuli in each trial. For the same reason, the appearance and relative positions of the shapes varied from trial to trial. The interested reader is referred to the original paper of Avarguès-Weber et al. (2012) for more details.

To assess the quality of the honeybees' concept learning, four different transfer tests 
were performed. The layout of the tests and the results obtained are presented in Figure 4. During the experiments, half of the bees were trained with the above-below relation and the other half were trained with the left-right relation. The sameness-difference concept was always represented by two different colors or two different patterns during the trials. The first transfer test showed that the bees could successfully apply previously rewarded spatial relations to unseen stimuli. For example, the group trained with achromatic figures was presented with patterns based on colored circles (Figure 4). The second and third transfer tests showed that bees, presented with stimuli having the same spatial relation (either right or wrong) preferred stimuli corresponding to the learned sameness-difference concept. The final test revealed that both concepts are equally important in the learned experience. In other words, the bees' choices were random when confronted with stimuli that matched their learned spatial concept but conflicted with the learned difference concept. Thus, the bees were simultaneously applying both concepts.

\section{Fundamentals of Vector Symbolic Architecture}

As mentioned previously, VSAs are tool for encoding and performing operations on distributed representations of knowledge. They have previously been used mainly in the area of cognitive computing and natural language processing, as described by Kanerva (2009), Plate (2003) and Gallant and Okaywe (2013). The fundamental difference between the distributed and traditional (localist) representations of data is that in localist computing architectures each bit and its position within a structure of bits are significant (for example a field in a database has a predefined offset amongst other fields, and a symbolic value has a unique representation in ASCII codes) whereas in a distributed representation all entities (and structured combinations of entities) are represented by vectors of very high dimensionality. That is, entities are represented by the direction of a vector in a high-dimensional space and every bit contributes to defining the vector's direction. In a localist representation, single bits or small groups of bits can be interpreted without reference to the other bits. In a distributed representation it is only the total set of bits that can be interpreted. For the remainder of the article, the term HD-code is used when referring to such codes. The prefix HD stands for High Dimensionality, and refers to that fact that in HD-codes, several thousand positions (of binary numbers) are used to represent a single entity; Kanerva (2009) proposed the use of vectors comprising 10000 binary elements. Such entities have a number of useful properties that are discussed in detail below.

\subsection{Randomness}

Randomness means that the values on each position of an HD-code are independent of each other, such that " 0 " and " 1 " components are equally probable. In very high dimensional systems, the distances from any arbitrary chosen HD-code to more than $99.99 \%$ of all other vectors in the representation space are concentrated at around 0.5 normalized Hamming distances. Interested readers are referred to Kanerva (2009) and Kanerva (1988) for a comprehensive analysis of the probabilistic properties of the hyperdimensional representation space. 
In VSAs, information is encoded in the relations (angles) between vectors rather than the vectors' absolute directions. Thus, the choice of vectors representing 'atomic' entities is arbitrary. If the atomic entities should be unrelated (because there are no initially learned relations, one should always assume the entities to be unrelated in order to avoid bias), the HD-codes representing them should be chosen to be mutually orthogonal (maximally dissimilar). In high-dimensional spaces, two vectors chosen at random are very likely to be near-orthogonal. Thus, the desired properties can be obtained simply by randomly selecting the vectors used to represent atomic entities.

\subsection{Similarity metric}

The similarity of two binary representations is characterized in terms of the normalized Hamming distance, which (for two vectors) measures the number of positions in which the vectors differ, divided by their size:

$$
\Delta_{H}(A, B)=\frac{1}{d}\|A \oplus B\|_{1}=\frac{1}{d} \sum_{i=0}^{d-1} a_{i} \oplus b_{i},
$$

where $a_{i}, b_{i}$ are bits at position $i$ in vectors $A$ and $B$ of dimension $d$, and $\oplus$ denotes the bit-wise XOR operation.

\subsection{Bundling of Vectors}

The bundling operation is used to join multiple entities into one structure; it is implemented by a thresholded sum of the HD-codes representing the entities. A bitwise thresholded sum of $n$ vectors yields 0 when $n / 2$ or more arguments are 0 , and 1 otherwise. If the sum produces an even number, the resulting tie is broken randomly. This is equivalent to adding an extra random HD-code Kanerva (2009).

The terms "thresholded sum" and "majority sum" are used interchangeably in this work, and both refer to the sum $[A+B+C]$. The relevant properties of the majority sum are:

- the result is a random vector, i.e. the number of ' 1 ' components is approximately equal to the number of ' 0 ' components;

- the result is similar to all vectors included in the sum;

- as the number of HD-codes that are operands of the majority sum operator increases, the Hamming distance between the result of the operation and any of the operands tends to 0.5 (which is the expected similarity of any two HD-codes selected at random). That is, the information capacity of the result vector is finite and information about the operands is lost as more of them are combined.

\subsection{Binding of vectors}

Binding, which can be interpreted as assigning a value to a field, is implemented as a bit-wise XOR operation on the corresponding vectors. The important properties of the XOR operation are:

- the binding is invertible (unbinding), i.e. if $C=A \oplus B$ then $C \oplus A=B$; 
- the binding distributes over bundling $D \oplus[A+B+C]=[D \oplus A+D \oplus B+D \oplus C] ;$

- when the operands have a density of 0.5 (i.e. an equal number of 1 and 0 bits) the result has an expected density of 0.5 .

- the result is dissimilar to both operand vectors: $\frac{\|B \oplus(A \oplus B)\|_{1}}{d}=0.5$ and $\frac{\|A \oplus(A \oplus B)\|_{1}}{d}=$ 0.5. However, binding preserves relational similarity: $\Delta_{H}(A, B)=\Delta_{H}(C \oplus$ $A, C \oplus B)$.

\subsection{Composition and decomposition of structures in distributed representations}

Representing a compositional structure containing roles (fields) and fillers (values) to describe relations such as (1) requires both VSA operations: binding for the association of roles with fillers and bundling for joining the set of bound role-filler components. Thus, a compositional structure $S$ with three roles (fields) and associated fillers (values) is defined as: $S=\left[R_{1} \oplus F_{1}+R_{2} \oplus F_{2}+R_{3} \oplus F_{3}\right]$.

The extraction of information from distributively represented structures is the reverse of the encoding process. Namely, a particular vector-filler is extracted by XORing the HD-code representing the structure with the desired vector-role. This follows from the self-inverse property of binding with XOR.

To understand the process, consider the following example. To decode filler $F_{1}$ from compositional structure $S$, the following set of operations is performed on $S$ :

1. XOR is performed on the HD-code representing role $R_{1}$ to retrieve its filler: $F_{1}^{\prime}=S^{\prime} \oplus R_{1}=F_{1}+$ NOISE.

2. The noisy vector $F_{1}^{\prime}$ is passed to the item memory to find the best clean match, i.e. to search for an entry with that a minimal normalized Hamming distance to $F_{1}^{\prime}$.

Note that in the context of VSAs, NOISE is not the same as a random signal. In VSAs, everything (both atomic and composite) is represented by a vector. The direction of the vector represents the identity of the thing that it represents, and the magnitude of the vector represents the strength of the evidence for (or degree of belief in) the thing represented by the vector. The natural basis vectors (the dimensions) of the vector space have no intrinsic meaning. Therefore, there is nothing intrinsic to a vector to indicate whether it represents an atomic or complex object. The only way a VSA system can recognize a vector as representing a composite entity is by being able to decompose it into its components. This requires that the components have a special functional status in the system. This is done by providing a clean-up or item memory. By being stored in this memory, vectors gain a distinguished status, which means that the system will try to recognize arbitrary vectors as composites of the items in the clean-up memory. When performing an unbinding such as $A \oplus[A \oplus B+C \oplus D]=$ $[(A \oplus A) \oplus B+A \oplus C \oplus D]=[B+A \oplus C \oplus D]$ the operation will generate terms like $A \oplus C \oplus D$, which are legitimate vectors but do not correspond to any entity that the system would ever want to represent (whereas $B$ does). Therefore, terms like $A \oplus C \oplus D$ are noise in the sense that they do not correspond to a representation that the system would ever be interested in. They are filtered out by using a clean-up or 
item memory populated with vectors like $A, B, C$, and $D$. The clean-up memory can be interpreted in a number of ways. At one extreme, it can be regarded as a projection onto the subspace spanned by the items. This allows a vector to be cleaned-up to a weighted sum of the items in the memory. If the memory contained $A, B, C$, and $D$, the vector $[p A+q B+X+A \oplus B]$ would be cleaned up to $[p A+q B]$ (where $p$ and $q$ are scalar multipliers). At the other extreme there can be competition (equivalent to lateral inhibition) between the retrieved vectors so that only the item with the highest magnitude survives. In the example above, the result would be $[A]$ if $p>q$.

\subsection{Architectural implementation of VSA operations}

The algebra of VSA includes other operations, such as permutation of vectors. Since these operations are not relevant to this article, their properties are not described. The most important point to realize is that all these VSA operations (e.g. binding, bundling, composition, decomposition) are implemented architecturally. That is, they are mathematical and systematic consequences of simple arithmetic operations on vector spaces, which can be easily implemented through the pattern of connections between the neurons.

Given an appropriate pattern of neural connections, these operations will be applied uniformly and systematically to all representational vectors, even novel ones. This stands in contrast to the behavior of traditional backpropagation neural networks, which lack this property of systematicity and consequently cannot generalize an existing operation to novel inputs without extensive training.

\subsection{Learning by example in VSA-based systems}

The ability of HD-codes to learn mappings between concepts from examples was first demonstrated by Kanerva (2000). Similar ideas were later exploited by Emruli et al. (2013); Emruli and Sandin (2014). This ability stems from the self-inverse binding property of Binary Spatter Codes (and some other VSAs). $A \oplus B$ represents the binding of $A$ with $B$, but it can equally be interpreted as a representation of a mapping from $A$ to $B$ and vice versa. Binding $A \oplus B$ with $A$ yields $B$, hence, $A \oplus B$ can be interpreted as the representation of a mapping that when applied to $A$ transforms it to $B$. The mapping is bidirectional, so it can also transform $B$ into $A$. This bidirectionality can be avoided if necessary, but is outside the scope of this paper. Learning by example can be regarded as relational learning. In the scope of this paper, learning by example is a core functionality of the concept learning block and is based on the ability of VSAs to recognise the structural similarity of knowledge structures. In essence, the key component is a vector $\mathbf{M}$, which acts as a mapping structure for inferring similarity between compositional structures. Its functioning can be demonstrated by considering an example provided by Kanerva (2000). It is known, that if $a$ is the mother of $b$, then $a$ is the parent of $b$. Both data structures are represented using the approach from subsection 4.5. For the mother relation mother $A B=[$ mother $+\operatorname{mot} \oplus \mathbf{A}+\mathbf{c h i l d} \oplus \mathbf{B}]$, where mother is the HD-code representing the relation for the mother, mot and child are HD-codes for roles in this structure. For the parent relation parent $\mathbf{A}_{A B}=[$ parent $+\operatorname{par} \oplus \mathbf{A}+\mathbf{c h i l d} \oplus \mathbf{B}]$, where parent is the HD-code representing the relation for the parent, and par and child are HD-codes for roles in this structure. Vector $M$ in turn is constructed as the binding 
of parent $\boldsymbol{A}_{A B}$ and mother $_{A B}$, i.e. $\mathbf{M}=\mathbf{M}_{A B}=$ mother $_{A B} \oplus$ parent $_{A B}$. Thus the mapping vector is based on the example. The mapping vector is updated in a similar way as additional examples are encountered: $\mathbf{M}=\left[\mathbf{M}_{A B}+\mathbf{M}_{C D}+\mathbf{M}_{E F} \ldots\right]$, where $c, d, e, f$, are new examples of the same relations. Thus, it is possible to generalize that $x$ is the parent of $y$ given only that " $x$ is the mother of $y$ " in form mother m $_{X Y}$ by unbinding $\mathbf{M} \oplus$ mother $_{X Y}$ for previously unseen fillers $x$ and $y$, because the result of unbinding will be most similar to parent ${ }_{X Y}$. This behavior is due to the fact that the same literal $A$ has been present in both relations, $\operatorname{mot} \oplus A$ and $\operatorname{par} \oplus A$. The binding $M_{A B}$ contains terms like $(\operatorname{mot} \oplus A) \oplus(\operatorname{par} \oplus A)=\operatorname{mot} \oplus \operatorname{par}$, which implement the mapping of the roles independently of the new fillers.

The power of this approach is considerable. Unlike learning in classical neural networks, this method does not require thousands of training episodes to slowly adjust synaptic weights. Instead, the VSA mapping vector can be learned from a few examples, each seen only once. Each example constructs a representation in a single feed-forward pass through the network and the few examples are accumulated in a short-term memory system. Furthermore, the VSA mapping vector can be successfully applied to novel relational examples. The only requirement is that the atomic roles and fillers are already stored in the clean-up memory.

In this work, learning by example is used by the processing unit to learn concepts. Mapping structures for different concepts are used to store the bindings of the representations of reward/punishment and HD-codes representing episodes. New precedents identified for a particular concept are added to the corresponding mapping vector. An illustrative example demonstrating concept formation and processing is presented in the next section.

\subsection{Anchoring the proposed VSA-based online concept learning architecture to psy- chological models of similarity}

The analysis of similarity is a mature psychological discipline with a set of established models. The goal of this section is not to formally prove the strict correspondence of the VSA-based concept learning pipeline to the general form of analogical similarity. Rather the intention is to demonstrate that the proposed VSA-based concept learning approach exhibits some characteristic properties of analogical similarity models, which makes it suitable for functional modeling of bees' concept-learning process. Three main classes of similarity model exist: spatial models, featural models and structural mapping. Markman and Gentner (2005) argue that all three models could be used in combination for holistic modeling of cognition.

The following discussion introduces the main postulates of each model class. In spatial models (Shepard, 1962; Nosofsky, 1986) concepts are represented as points or vectors in a semantic space. Similarity is assessed by finding the distance between points using some scalar metric. The main advantage of this model is its low computational complexity; its main drawback is that it is impossible to access the specific commonalities and differences that form the basis of the similarity judgment.

The contrast model, which is also called the featural approach (Tversky, 1986), overcomes the shortcomings of spatial models by representing entities as sets of features. Similarity judgments are made by comparing pairs of features using elementary set operations. The intersection of sets of features contains commonalities; features 
outside the intersection are regarded as differences. Calculations of similarity in contrast models are computationally inexpensive: each feature in the representation of one item simply needs to be matched against the set of features representing the other item.

A third approach to similarity called structural mapping Gentner (1983) accounts for the importance of relations in similarity and seems to most closely match the similarity-judging process of the human brain. In models of this class, the representations consist of entities, attributes and relations. Relations are representational elements that relate two or more arguments, e.g. an above $(x, y)$ relation represents the above-below relation between objects $x$ and $y$. To compare pairs of relational representations, the representations must comply with the system's structural consistency constraints, namely the constraints of parallel connectivity and one-to-one mapping. Parallel connectivity means that if two relations are to be compared, their arguments must match. For example, if two above $(x, y)$ relations are compared then things on top and things on the bottom must be matched. One-to-one mapping means that each element in one representation matches to at most one element in the other. For example, if two relations (for instance, two above-below relations) are compared and both contain the same objects in conflicting states (e.g. if the first relation features a square in the 'above' position while the second relation has the same square in the 'below' position) then these two objects should be treated as different in the above-below test.

The structure mapping model is much more computationally intensive than spatial or contrast models. The calculation of parallel connectivity requires checking each correspondence among relations to ensure that the arguments of those relations also match. In addition, enforcement of the one-to-one mapping constraint may require many comparisons between potential matches. While the three model types differ in their expressive power, Markman and Gentner (2005) argues that combinations of these classes could potentially be used to capture different aspects of cognitive processing. For example, some combination of spatial and contrast models could be used for fast judgments of the degree of similarity while structural mapping could be used for slower, more computationally expensive but richer comparisons that give access to the commonalities and differences of the compared objects. Psychological studies have provided evidence that humans use both modes of assessment: computationally simpler forms of similarity matching operate continuously, searching for similarities among items in the environment and between items in the environment and background knowledge, and the results of these simple comparisons are used to guide the allocation of resources for more detailed similarity analysis.

\subsection{Comparing structural mapping similarity models to the VSA-based online learn- ing architecture}

A detailed analysis of the correspondence between the proposed VSA-based concept learning approach and general models of analogical similarity would be beyond the scope of this article. In lieu of such a discussion, this section highlights some important parallels between the main postulates of the three known classes of analogical similarity model and the VSA-based architecture proposed in this work. Before starting, it is important to be aware of the dualism of distributed representations and Vector Symbolic Architectures. On the one hand, a VSA is a tool for encoding structured 
information as discussed in Section 4. On the other hand, the usage of a distributed encoding approach (i.e. high-dimensional binary codes with structures being represented by basic binary arithmetic operations) enables comparisons of structured information using a scalar metric (the Hamming distance).

This dualism leads to the first parallel between the proposed approach and the psychological theories of similarity, which is that while VSA could potentially (to a certain extent) be used for structural mapping analysis it also exhibits some functional properties of spatial similarity models. The comparative representation of relations (2) is used to encode the presence of feature-based similarities or differences in the represented episode in a comparable way to that envisaged by contrast similarity models. The similarities and differences between features are detected by the feature extraction block using elementary comparison operations. The resulting comparative relations can be used either by themselves or in combination with the bonding representations (1) to facilitate rapid evaluation of the observed episode. The latter usage is adopted in the proposed online concept learning system.

Note that both spatial and featural similarity models implicitly assume low-dimensional spaces and are therefore not strictly mappable to cases involving high-dimensional spaces. However, a functional parallel can be drawn: since the VSA-encoded relations are essentially points in the high-dimensional space of binary distributed representations, their similarity can be quickly assessed by computing a scalar distance metric. If the vectors representing the stimuli are scattered over a non-trivial subspace of the total vector space, their similarity will only be discriminable to the resolution of same-different, whereas if the vectors are constrained to fall in a sufficiently lowdimensional subspace then it will be possible to compute their similarity as a graded quantity. The notion of relations is central to both the proposed system and the structural mapping theory. The outcome of the initial processing of the episode is that the feature extraction block determines the entities, their attributes and relational properties. Note that the structural similarity theory relies on subgraph isomorphism between the two representations to determine the correspondences between entities and relations. A weaker form of this theory would use the literal similarity of relational roles to determine the correspondence between fillers. The proposed system suggests two kinds of templates for relations: bonding and comparative. Bonding representations are used to represent proper directional relations - for example, the relation bond (above - below, $x, y)=[$ above - below + above $\oplus x+$ below $\oplus y]$ encodes the relation "entity $x$ is above entity $y$ ". Representing relations in this way ensures that both the parallel connectivity and the one-to-one mapping constraints are satisfied. Compliance with these constraints is ensured by the way the representations are used, i.e. by binding them together.

Given the self-inverse-binding property of high-dimensional binary spatter codes, the repeated literal values serve to "align" the corresponding elements. In a fully general analogical mapping model, however, both the fillers and the roles may differ between the structures being compared, so this alignment mechanism would not be sufficient. Parallel connectivity is ensured by the comparative inclusion of the directionality in the VSA representation of the relation. That is, for the (detailed) comparison of two relations of the same type, one must first unbind in both relations the HD-codes for the objects located above (by performing an XOR operation with the HD-code for above) 
and then unbind the HD-codes for the objects located below (by performing an XOR operation with the HD-code for below), and finally compare the results pairwise, i.e. above with above and below with below ${ }^{2}$. To demonstrate the functional satisfaction to the one-to-one mapping constraint, suppose the system encountered another relation of the same type but where object $x$ is located below another object, say $z$, i.e. bond (above - below, $z, x)=[$ above - below + above $\oplus z+$ below $\oplus x]$. Obviously, although the HD-codes for object $x$ are the same in both cases, the outcome achieved by encoding the relations would be different because binding $x$ with a codeword for below would produce a dissimilar result to that obtained when binding $x$ with a codeword for above due to the mutual dissimilarity of above and below. Note again that this discussion should not be interpreted as a direct correspondence to the one-to-one mappings discussed in the analogical mapping literature, in which this constraint refers to mapping between fillers. That is to say, the constraint is that if filler $x$ maps to filler $y$ in one part of the mapping, it should do so everywhere in the mapping. In the pipeline proposed herein, the role-filler binding is conceived as a mapping, but in strict terms it is a different type of mapping to the filler-filler mappings discussed in analogical mapping theory.

In summary, the form used to represent relations in the VSA (1) and (2) as well as the representation of the episode (3) were chosen because:

1. There is experimental evidence that the target biological system (honeybees) is capable of both feature-based and relation-based similarity analysis;

2. These forms facilitate quick holistic similarity assessment based on a well-defined scalar metric of similarity for the target decision making scenario (selecting one of several alternative paths).

\section{Design of the online concept learning system}

This section describes the design of the online VSA-based concept learning system and exemplifies its current implementation by replicating the honeybee experiments described in Section 3.1. Table 1 introduces the notation used throughout this section.

\subsection{Vision circuitry}

The first block of the system is the vision circuitry, which perceives the episode and extracts the features of the presented visual stimulus. To facilitate further discussion, the set of considered stimuli was restricted to that used in the honeybee experiments, i.e. geometrical figures (circles) with a single-colour fill (see Figure 5). Without claiming any biological plausibility, the feature extraction block is implemented using the standard image processing toolbox; in the current implementation, feature extraction was implemented using the regionprops() function in MATLAB. This function takes a binary image as a logical array and then measures and outputs a set of properties for

\footnotetext{
${ }^{2}$ Note that this way of using the representations would involve a lot of serial computation; the example was chosen mainly to demonstrate that the proposed approach functionally satisfies the requirement of parallel connectivity. One of the big advantages of a VSA is that it enables holistic computation.
} 
Table 1: Notations used in Section 5

\begin{tabular}{|l|l|}
\hline Symbol & Definition \\
\hline ab & $r e$ for the above-below relation \\
\hline a & $r o_{1}$ for the 'above' role \\
\hline b & $r o_{2}$ for the 'below' role \\
\hline Is & Result of comprel(f) for the larger-smaller relation \\
\hline sd & Result of comprel(f) for the sameness-difference relation \\
\hline equal_size & HD-code representing equality in the larger-smaller feature-relation (result of comparison) \\
\hline different & HD-code representing difference of objects in the same-different feature-relation (result of comparison) \\
\hline EXPERIENCE & mapping vector for concept learning \\
\hline REWARD & HD-code for reward (sucrose) \\
\hline Cy & Encoded values of features for a yellow circle \\
\hline Cp & Encoded values of features for a purple circle \\
\hline STIMULUS & Representation of the $i$ th stimulus \\
\hline
\end{tabular}

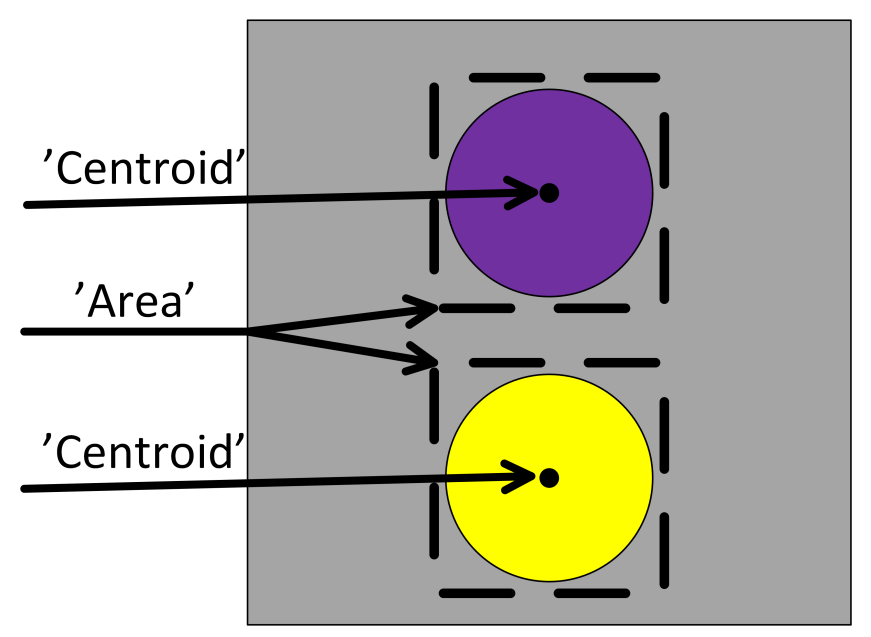

Figure 5: An example of a visual stimulus and the output of the vision circuitry, implemented using the regionprops() function of MATLAB.

each object in the episode. Output parameters produced by this function that are relevant to this discussion include 'Area' and 'Centroid', which are used to extract higher level features such as shapes, colours, raw relations (relative spatial placement, relative sizes, etc.). These outputs are then fed to the stimuli encoding block.

\subsection{Stimuli encoding block}

The system does not generally use human-comprehensible labels for relations. However, to facilitate interpretation, such labels are used in this section. For example if the 'Centroids' of two objects differ with respect to their position on the horizontal axis, the two objects are labeled as being in the left-right relation. Similarly, if the 'Centroids' of two objects differ with respect to their position on the vertical axis, they are labeled as being in the above-below relation. Similarly, objects are labeled as being 
in a sameness-difference relation on the basis of their colors or shapes at their 'Centroids'. Finally, pairs of objects are labeled as being in the larger-smaller relation on the basis of their 'Area' values. Note, that a very specific symbolic processing has been applied to the "perceptual" output of regionprops(). Given the experimental design of the visual stimuli, it was possible to predict the encoding of the stimuli from the experimental conditions with perfect accuracy, and only the minimal features required to represent the stimuli were encoded. Consequently, the visual component of regionprops() does not form part of this paper's contributions, and no attempt was made to investigate any visual stimuli that might interfere with or adversely affect the encoding in any way.

Next, the stimuli encoding block generates random HD-codes to encode the objects of the episode and all of its features. These vectors are stored in the item memory. As the system continues to operate new HD-codes are added to the item memory for each newly detected feature or an object. The representations for repeated items are, therefore, taken from the item memory.

Next, for each new feature-relation and the objects engaged within it, one VSA template is drawn from the pool to encode the relation as a VSAs representation. This step is best explained with reference to a concrete example, for which we have chosen the encoding of the episode depicted in Figure 5. In this case, the vision circuitry will detect two objects of the same shape (circles) and size. It will also detect that the objects' colours are different and that the purple circle is located above the yellow one. Assuming that the system's item memory is initially empty, the stimuli encoding block will generate HD-codes as summarized in Table 1.

The following VSA-relations are encoded:

1. $R E L 1=[\mathbf{a b}+\mathbf{a} \oplus \mathbf{C p}+\mathbf{b} \oplus \mathbf{C y}]$. This is the encoded above-below relation between objects with IDs $\mathbf{C p}$ (purple circle) and $\mathbf{C y}$ (yellow circle) using a bonding template;

2. $R E L 2=[\mathbf{l s} \oplus$ equal_size $]$. This is the encoded larger-smaller relation using a comparative template;

3. $R E L 3=[\mathbf{s d} \oplus \mathbf{d i f f e r e n t}]$. This is the encoded sameness-difference relation using a comparative template;

When all relations are represented in VSA format, the encoding block constructs a VSA representation of the entire episode, which is called EPISODE as in (3). In the case of the considered example, the distributed representation generated from the episode in Figure 5 is $\mathbf{E P I S O D E}_{1}=[\mathbf{a b}+\mathbf{a} \oplus \mathbf{C p}+\mathbf{b} \oplus \mathbf{C y}+\mathbf{l s} \oplus \operatorname{similar}+\mathbf{s d} \oplus$ different]. When the episode's representation is constructed, it is temporarily stored by the stimuli encoding block, awaiting the arrival of the reward signal to complete the process. Note that a given reward could be associated with many episodes. In such cases, the system should store all of them in the temporal memory. This aspect is implementation-specific and as such the details of the process are not discussed further. In the considered example based on the honeybee experiments, both episodes were involved in the reasoning about the reward.

When the reward signal arrives, a VSA representation of the present experience is constructed as EXPERIENCE $_{p \mid n}=\left[\right.$ EXPERIENCE $_{p \mid n}+$ EPISODE $_{i} \oplus$ REWARD $\left._{p \mid n}\right]$. For example, if three stimuli are provided, the representation will be of the form 
EXPERIENCE $_{p \mid n}=\left[\right.$ EPISODE $_{1} \oplus$ REWARD $_{p \mid n}+$ EPISODE $_{2} \oplus$ REWARD $_{p \mid n}+$ EPISODE $_{3} \oplus$ REWARD $\left._{p \mid n}\right]$. This ensures that HD-variable EXPERIENCE accu-

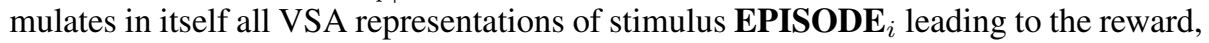
which is encoded by HD-code REWARD $\mathbf{R}_{p \mid n}$. Here, the index " $p \mid n$ " indicates either a positive or a negative reward. Note that the EXPERIENCE vector plays the same role as the mapping vector $\mathbf{M}$ in subsection 4.7. The distributed representation of the experience is then stored in the associative memory for later recall (i.e. reasoning).

In general the system can store experiences for different types of reward in separate EXPERIENCE vectors, one for each reward type. Different learning strategies could be designed based accounting for a give episode relative weights of different rewards. The simplest of the strategies is to favor only one type of reward. Whether multi-reward learning strategies are the case for the cognitive behavior of the honey bees is yet to be understood, therefore, in this article the artificial learning system stores only positive experience.

\subsection{Concept recall (reasoning)}

The system implements reasoning via the recall of an EXPERIENCE stored in the associative memory. Recall can be performed as soon as the first reward has been recorded. In the recall process, the processing unit first receives representations for two scenes and constructs relational representations for each one as in (3). For reasons of consistency, the representations of the scenes in the recall phase will also be called episodes and denoted as EPISODE ${ }_{1}^{*}$ and EPISODE $_{2}^{*}$, respectively. The superscript “*” indicates that the episodes may be being presented to the system for the first time. The system then retrieves the current EXPERIENCE from the associative memory, after which it unbinds the representations of the stimuli from EXPERIENCE as:

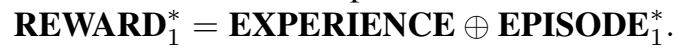

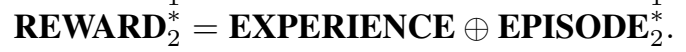

Finally, the processing unit chooses the episode whose normalized Hamming distance is closest to the target REWARD. In other words, it identifies that episode as the one most likely to provide a reward on the basis of past experience.

\section{Simulation and results}

The goal of the simulations was to assess the accuracy with which the system recalled the learned concepts. Because evaluating the performance of the vision circuitry would be outside the scope of this article, simulations were performed using 120 visual episodes similar to those used in the original honeybee experiments (Avarguès-Weber et al., 2012) (see Figure 4). Each episode was preprocessed to ensure that the stimuli were encoded exactly as expected in their design.

It should be emphasized, however, that the system does not attempt to model what is actually happening in the bee's brain. The architecture and its parameters are not constrained by knowledge about honeybees; the only bee-related fact used in their design was the knowledge that bees can encode and use multiple relational descriptions of a given episode. Thus, one should not expect the results to agree quantitatively with the bees' behavior for all possible levels of the visual problems that bees can solve. 
In the simulations, each object was not represented by a VSA representation incorporating all individual features. Instead, the whole object, e.g. a purple colored circle of size $s$, was encoded by one randomly generated HD-code. The dimensionality of the HD-codes in the simulations was 10000 elements ${ }^{3}$. In total, 25 HD-codes were generated to encode colored circles and the features-relations in each episode: 12 to represent objects (colored shapes); another 12 to represent the relations' templates ( 3 for above-below, 3 for left-right, 3 for large-small, and 3 same-different relations); and 1 to represent the positive reward signal.

The objective of the simulations ${ }^{4}$ was to evaluate the accuracy of concept recall after the same number of trials as in the real-world experiments, i.e. 30. For this purpose, all possible partial permutations of $6 \mathrm{HD}$-codes representing objects of 2 distinct HD-codes were presented during the training phase, giving 30 permutations in total. All 2-permutations of the remaining 6 objects were used to generate 30 test cases (60 episodes) in order to evaluate the accuracy of concept learning. During each round of the training process the system was presented with two episodes comprising the same objects arranged in different spatial relationships. The environment generated two reward signals, one positive and one negative. The positive reward corresponded the situation where the bee encountered sucrose at the end of the tunnel, the negative reward corresponded to finding a quinine solution. Only positive rewards were stored in the EXPERIENCE VSA in the associative memory. For the simulations, it was decided that the positive REWARD would be associated with the above-below relation. The associative memory was empty in the beginning of the simulation. After training the system with 30 training trials, the accuracy of the VSA-based concept learning process was tested using 30 new previously unseen cases. In the testing phase, two VSA-encoded episodes were used to unbind the REWARD representation for each episode from the EXPERIENCE VSA recalled from the associative memory. The results of the unbinding operation (two noisy versions of the REWARD) were compared with the clear version of REWARD representing the positive reward, which is stored in the item memory. The system chooses the episode having the smallest normalized Hamming distance to the clean REWARD representation. During the testing phase, the learning system was disabled.

\subsection{Results of the transfer test simulations}

Four simulations as described above were conducted, using the four types of transfer tests described in Section 3.1.

The results of the four transfer test simulations are presented in Figure 6, which shows the normalized distributions of the normalized Hamming distances from REWARD $\mathbf{R}_{1}^{*}$ and REWARD ${ }_{2}^{*}$ to REWARD for 30 different testing trials. The results are clearly positive in that the concept recall accuracy in all four simulations resembles the results of the real-world experiments very closely. In particular, in transfer tests one, two and three the system recognized the correct concept in all of the test trials, which

\footnotetext{
${ }^{3}$ This is a typical dimensionality for VSA simulations; for example, see Kanerva (2009).

${ }^{4}$ The MATLAB scripts for the experiments are available as supplementary materials as well as via https://www.dropbox.com/s/xlfulqdmkm15sf1/Experiments. $z i p$ ?dl=0.
} 

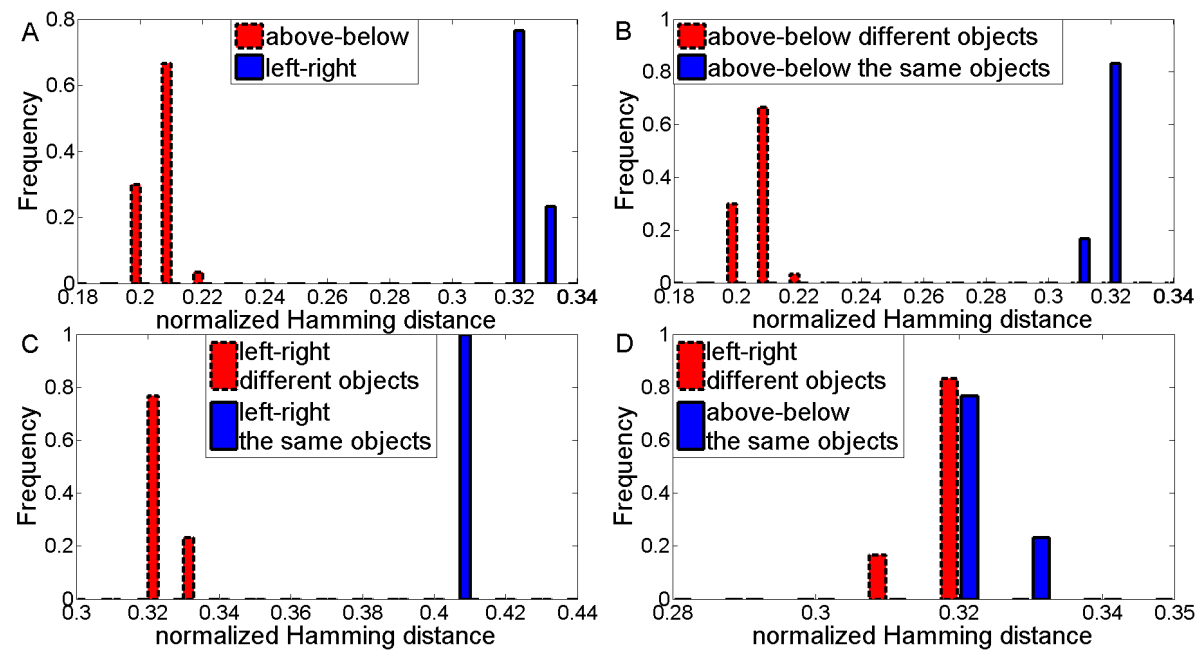

Figure 6: The results of the transfer test simulations. Results are presented in the form of histograms for 30 trials displaying the frequency of normalized Hamming distances to the sucrose reward for the offered alternatives. In all simulations of transfer tests, the system learned the above-below concept. A. Simulation of transfer test 1 . The distribution of distances indicates a preference for stimuli showing the above-below concept. The results are consistent with those of transfer test 1 in Figure 4. Mean normalized Hamming distance for above-below trials: $0.2077 \pm 0.0039$; for left-right trials: $0.3219 \pm 0.0037$. B. Simulation of transfer test 2. The distribution of distances indicates the preference for stimuli with different objects. The results are consistent with those for transfer test 2 in Figure 4. Mean normalized Hamming distance for trials with different objects: $0.2071 \pm 0.0039$; for trials with the same objects: $0.3186 \pm 0.0032$. C. Simulation of transfer test 3 . The distribution of distances indicates the preference for stimuli with different objects. The results are consistent with those of transfer test 3 in Figure 4. Mean normalized Hamming distance for trials with different objects: $0.3219 \pm 0.0037$; for trials with the same objects: $0.4137 \pm 0.0014$. D. Simulation of transfer test 4 . The distribution of distances for trials with two stimuli are extremely close, i.e. there is no preference for any of the stimuli. The results are consistent with those of transfer test 4 in Figure 4. Mean normalized Hamming distance for left-right trials with different objects: $0.3219 \pm 0.0037$; for above-below trials with the same objects: $0.3186 \pm 0.0032$.

is consistent with the results of the real-world experiments. The simulation for the fourth yielded interesting results: the distributions of normalized Hamming distances to REWARD were very close, to the point that they intersected. Consequently, the random variation in the normalized Hamming distances determined which stimulus was chosen. This behavior is equivalent to the random choice between stimuli observed in the real-world experiments under the corresponding conditions.

\section{Discussion and Conclusion}

This article presented an artificial online concept learning system that imitates part of the cognitive behavior of a living organism - concept learning by honey bees. The system uses Vector Symbolic Architectures to implement the representation of an episode, the associative memory and the reasoning. The proposed design for the online learning system is generic in the sense that it allows reasoning based on any relations 
that are discovered at the system's runtime. The main achievements presented in this work are:

- Vector symbolic architectures were used to represent structured combinations of features/relations and to apply those relations to novel structures in an online learning context;

- The choice of the relation represesentation was motivated by classical analogical similarity models;

- VSA representations of relations formed a central part of the learning pipeline with a perceptual circuitry and made it possible to apply those relations to novel structures without requiring the massive training needed for classical neural networks;

- A proof-of-concept validation of the proposed online learning pipeline was presented based on the concept learning capabilities observed for honeybees.

The proposed model is definitely biologically inspired. While its biological plausibility is, of course, a subject for future investigation, the choice of the VSA format for relation representation was tailored to the specifics of the real-world experiments with living organisms that inspired our study. The experiments demonstrated honeybees' ability to recognize the properties of a pair of objects as well as the relations in which they are engaged. Further work will be required to determine whether the capabilities revealed in these experiments reflect the full extent of the bees' cognitive capacities. However, for the "level of cognition" established to date, the presented system imitates real-life outcomes very closely. This is a very positive result and constitutes a definite step towards closing the gap between VSAs, small brains and neuromorphic computing that was identified by Sandin et al. (2014) .

To model the concept learning process of organisms with higher cognitive capabilities (i.e. the ability to analyze episodes with more than two objects), more research will be needed to design suitable VSA templates for the online creation of VSAs encoding the relations between multiple objects. Preliminary efforts along these lines have been initiated; for now it is worth noting that there is reason to believe it will be possible to develop such templates because it has been demonstrated that even higher animals can only focus on a limited number of objects simultaneously. This means that the number of templates required for the analysis of even very complex episodes is finite.

This article focused on the proof-of-concept validation of the applicability of VSAs for imitating the learning behavior of living organisms. The main claim of this article is that there is a class of simple artificial systems that do not require the implementation of computationally intensive cognitive architectures (e.g. those based on the usage of Sparse Distributed Memory Kanerva (1988)). In certain applications, rather advanced cognitive behavior can be implemented using only simple techniques.

Another important aspect of this work, which is currently under investigation, is the time performance of the artificial online learning system. Specifically, it is interesting to investigate different approaches to reward collection and the time required to retrain the system to recognize new concepts. On the implementation side, the proposed 
architecture has very promising performance properties since all reasoning is done by simple arithmetic operations on binary codewords.

The claim of this article about VSAs having more superior learning efficiency (time to learn) than the traditional machine learning techniques is based on their main distinctive property - the on-the-fly (one-shot) learning (Rachkovskij and Kussul, 2001). While all traditional schemes require more than one learning cycle the question of how much more efficient the VSAs learning curve is is important to address by proper benchmarking. This comparison is a part of the future work.

The vision circuitry used in this work was implemented using traditional image processing algorithms. In addition, the content of the episodes was static, i.e. did not change over time. However, these limitations are not fixed; there is definitely the potential to create more bio-plausible solutions such as neuromorphic vision systems (Sandin et al., 2014). When modeling higher level cognition, it is important for the system to be able to capture motions and the temporal context as objects in the episode's analysis. A related challenge is the problem of encoding episodes with real-world objects. A possible way to include episode dynamics in the analysis is to use a longterm memory model for sequence storage such as Sparse Distributed Memory (SDM) Kanerva (1988) or other similar approaches, e.g. Rutledge-Taylor et al. (2014).

\section{Acknowledgements}

This work is partially supported by the Swedish Foundation for International Cooperation in Research and Higher Education (STINT), institutional grant IG2011-2025.

\section{References}

Aerts, D., Czachor, M., De Moor, B., 2009. Geometric analogue of holographic reduced representation. Journal of Mathematical Psychology 53, 389-398.

Avarguès-Weber, A., de Brito Sanchez, M. G., Giurfa, M., Dyer, A. G., 2010. Aversive reinforcement improves visual discrimination learning in free flying honeybees. PLoS ONE 5 (10), 1-11.

Avarguès-Weber, A., Dyer, A. G., Giurfa, M., 2011. Conceptualization of above and below relationships by an insect. Proceedings of the Royal Society B: Biological Sciences 278, 898-905.

Avarguès-Weber, A., Dyer, A. G., Combe, M., Giurfa, M., 2012. Simultaneous mastering of two abstract concepts with a miniature brain. Proceedings of the National Academy of Sciences 109, 7481-7486.

Avarguès-Weber, A., Giurfa, M., 2013. Conceptual learning by miniature brains. Proceedings of the Royal Society B: Biological Sciences 280, 1-9.

Avarguès-Weber, A., d'Amaro, D., Metzler, M., Dyer, A. G., 2014. Conceptualization of relative size by honeybees. Frontiers in Behavioral Neuroscience 8, 1-8. 
Avarguès-Weber, A., Dyer, A. G., Ferrah, N., Giurfa, M., 2015. The forest or the trees: preference for global over local image processing is reversed by prior experience in honeybees. Proceedings of the Royal Society B: Biological Sciences 282.

Dyer, A. G., Ravi, S., Garcia, J. E., 2014. Flying in Complex Environments: Can Insects Bind Multiple Sensory Perceptions and What Could Be the Lessons for Machine Vision? Journal of Software Engineering and Applications 7, 406-412.

Eliasmith, C., 2013. How to Build a Brain: A Neural Architecture for Biological Cognition. Oxford University Press.

Emruli, B., Gayler, R. W., Sandin, F., 2013. Analogical mapping and inference with binary spatter codes and sparse distributed memory. In: The proceedings of the international joint conference on neural networks, pp. 1-8.

Emruli, B., Sandin, F., 2014. Analogical mapping with sparse distributed memory: A simple model that learns to generalize from examples. Cognitive Computation 6 (1), 74-88.

Emruli, B., Sandin, F., Delsing, J., 2014. Vector space architecture for emergent interoperability of systems by learning from demonstration. Biologically Inspired Cognitive Architectures 9, 33-45.

Gallant, S. I., Okaywe, T. W., 2013. Representing objects, relations, and sequences. Neural Computation 25 (8), 2038-2078.

Gayler, R. W., 1998. Multiplicative binding, representation operators \& analogy. In: Gentner, D., Holyoak, K. J., Kokinov, B. N. (Eds.), Advances in analogy research: Integration of theory and data from the cognitive, computational, and neural sciences. New Bulgarian University, Sofia, Bulgaria, pp. 1-4.

Gayler, R. W., Levy, S. D., 2009. A distributed basis for analogical mapping. In: The Proceeding of the second International Conference on Analogy, 2009. pp. 1-10.

Gentner, D., 1983. Structure-mapping: A theoretical framework for analogy. Cognitive Science, 7, 155-170.

Giurfa, M., Zhang, S., Jenett, A., Menzel, R., Srinivasan, M. V., 2001. The concepts of "Sameness" and "Difference" in an insect. Nature 410, 930-933.

Kanerva, P., 1988. Sparse Distributed Memory. The MIT Press.

Kanerva, P., 1997. Fully distributed representation. In: Real World Computing Symposium. Vol. 97. pp. 358-365.

Kanerva, P. 2000. Large patterns make great symbols: An example of learning from example. In: Stefan Wermter and R. Sun (Eds.). Hybrid neural systems, Vol. 1778, pp. 194-203. 
Kanerva, P., 2009. Hyperdimensional computing: an introduction to computing in distributed representation with high-dimensional random vectors. Cognitive Computation 1 (2), 139-159.

Kleyko, D., Osipov, E., 2014. Brain-like classifier of temporal patterns. In: The Proceeding of the 2nd International Conference on Computer and Information Sciences - ICCOINS, 2014. pp. 1-6.

Kleyko, D., Osipov, E., 2014. On Bidirectional Transitions between Localist and Distributed Representations: The Case of Common Substrings Search Using Vector Symbolic Architecture. Procedia Computer Science 41, 104-113.

Kleyko, D., Osipov, E., Senior, A., Khan, A. I., Sekercioglu Y. A., 2015. Holographic Graph Neuron: a Bio-Inspired Architecture for Pattern Processing. Preprint at http://arxiv.org/pdf/1401.0742v1.pdf.

Kleyko, D., Osipov, E., Papakonstantinou, N., Vyatkin, V., Mousavi, A., 2015. Fault Detection in the Hyperspace: Towards Intelligent Automation Systems. In: The Proceeding of the 13th IEEE International Conference on Industrial Informatics - INDIN, 2015. pp. 1-6.

Levy, S. D., Bajracharya, S., Gayler, R. W., 2013. Learning Behavior Hierarchies via High-Dimensional Sensor Projection. In: The Proceeding of the Twenty-Seventh AAAI Conference on Artificial Intelligence, 2013.

Levy, S. D., Lowney, C., Meroney, W., Gayler, R. W., 2014. Bracketing the Beetle: How Wittgenstein's Understanding of Language Can Guide Our Practice in AGI and Cognitive Science. In: Artificial General Intelligence, Lecture Notes in Computer Science Vol. 8598, 73-84.

Markman, A., Gentner, D., 2005. Nonintentional similarity processing. The New Unconscious. Oxford University Press. 107-137.

Miller, G. A., 1956. The magical number seven, plus or minus two: Some limits on our capacity for processing information. Psychological Review 63 (2), 81-97.

Newell, A., 1994. Unified Theories of Cognition. Harvard University Press.

Nosofsky, R., 1986. Attention, similarity and the identification-categorization relationship. Journal of Experimental Psychology: General, 115(1), 39-57.

Plate, T. A., 2003. Holographic reduced representations: Distributed representation for cognitive structures. Center for the Study of Language and Information (CSLI).

Rachkovskij, D. A., Kussul, E. M., 2001. Binding and normalization of binary sparse distributed representations by context-dependent thinning. Neural Computation 13 (2), 411-452.

Rachkovskij, D. A., Kussul, E. M., Baidyk, T.N., 2013. Building a world model with structure-sensitive sparse binary distributed representations. Biologically Inspired Cognitive Architectures 3, 64-86. 
Rasmussen, D., Eliasmith, C., 2011. A Neural Model of Rule Generation in Inductive Reasoning. Topics in Cognitive Science 3 (1), 140-153.

Rutledge-Taylor, M. F., Kelly, M. A., West, R. L., Pyke, A. A., 2014. Dynamically structured holographic memory. Biologically Inspired Cognitive Architectures 9, 932.

Räsänen, O., Kakouros, S., 2014. Modeling Dependencies in Multiple Parallel Data Streams with Hyperdimensional Computing. IEEE Signal Processing Letters 21 (7), 899-903.

Sandin, F., Khan, A. I., Dyer, A. G., Amin, A. H. M., Indiveri, G., Chicca, E., Osipov, E., 2014. Concept Learning in Neuromorphic Vision Systems: What Can We Learn from Insects? Journal of Software Engineering and Applications 7, 387-395.

Shepard, R., 1962. The analysis of proximities: Multidimensional scaling with an unknown distance function, I. Psychometrika, 27(2), 125-140.

Snaider, J., Franklin, S., 2014. Modular Composite Representation. Cognitive Computation, 6(3), 510-527.

Soccol, D., Thurrowgood, S., Srinivasan, M. V., 2007. A vision system for optic-flowbased guidance of UAVs. In: Proceedings of the Ninth Australasian Conference on Robotics and Automation, 2007. pp. 1-7.

Srinivasan, M. V., 2006. Small brains, smart computations: Vision and navigation in honeybees, and applications to robotics. In: International Congress Series: 2nd International Conference on Brain-inspired Information Technology 1291, 30-37.

Srinivasan, M. V., 2010. Honey bees as a model for vision, perception, and cognition. Annual review of entomology 55, 267-284.

Srinivasan, M. V., 2011. Visual control of navigation in insects and its relevance for robotics. Current opinion in neurobiology 21, 535-543.

Srinivasan, M. V., Zhang, S., Altwein, M. and Tautz, J., 2000. Honeybee navigation: nature and calibration of the ÊżodometerÊij. Science 287, 851-853.

Tversky, A., Kahneman, D., 1986. Rational choice and the framing of decisions. Journal of Business, 59(4), S251-S278. 